\title{
Producing Anaglyphs from Synthetic Images
}

\author{
William Sanders, David F. McAllister \\ Department of Computer Science, North Carolina State University, Raleigh, NC USA 27695-8206
}

\begin{abstract}
Distance learning and virtual laboratory applications have motivated the use of inexpensive visual stereo solutions for computer displays. The anaglyph method is such a solution. Several techniques have been proposed for the production of anaglyphs. We discuss three approaches: the Photoshop algorithm and its variants, the least squares algorithm proposed by Eric Dubois that optimizes in the CIE color space, and the midpoint algorithm that minimizes the sum of the distances between the anagylph color and the left and right eye colors in CIEL*a* $\mathrm{b}^{*}$. Our results show that each method has its advantages and disadvantages in faithful color representation and in stereo quality as it relates to region merging and ghosting.
\end{abstract}

Keywords: anaglyph, CRT, LCD, Photoshop, CIE, RGB, ghosting, region merge, CIELab

\section{ANAGLYPHS}

Recent interest in virtual laboratories for distance learning applications have revived research in anaglyphs because the stereo image can be transmitted efficiently, inexpensive viewing devices can be used and several people can view the image simultaneously. Anaglyphs require the user to view the image with glasses having different color filters for each eye. The anaglyph color at a pixel is computed from the left eye color and the right eye color at the pixel. Most filters for electronic displays are designed so that the left eye filter blocks combinations of blue and green and the right eye filter blocks red. Blocking means that the color is seen as black or dark gray through the filter. In the discussion that follows, we use the red/cyan glasses No. 7003 from REEL3D (www.reel3d.com).

We assume no depth information in the scene is available. We consider some of the anomalies that may occur in creating anaglyphs from stereo pairs. We ignore the important issue of retinal rivalry ${ }^{2}$ which can create the appearance of ghosting. We will restrict our attention to CRTs, but the analysis is similar for other color displays that use the RGB color system.

\section{ANAGLYPH METHODS}

The set of representable colors or the color solid on a display using the RGB color system is the unit RGB cube (3cube). The cube lies in the 3 dimensional vector space $\mathbf{R 3}$. The basis or primaries are the colors red, green and blue.

The "RGB color solid" in the six dimensional vector space $\boldsymbol{R} \boldsymbol{6}$ is a unit hypercube (6-cube) with 64 vertices corresponding to the RGB corners of the cube in $R 3$ for the left and right eyes. Counting base 2 we can order the vertices of the 6-cube: $[0,0,0,0,0,0]=$ black, black $],[0,0,0,0,0,1]=[$ black, blue $], \ldots,[1,1,1,1,1,1]=[$ white, white $]$. Anaglyph methods compute a map from the 6-cube in $\boldsymbol{R} \boldsymbol{6}$ to $\boldsymbol{R 3}$. We can examine the order and placement of the image of the 6cube vertices to gain understanding of the method.

Three of the algorithms we analyze are linear; there is a 3 x 6 matrix representation of the map from $\boldsymbol{R} \boldsymbol{6}$ to $\boldsymbol{R} 3$ in each case. We define $v=\left[r_{l}, g_{l}, b_{l}, r_{r}, g_{r}, b_{r}\right]^{T}$ which are the RGB coordinates of the left and right eye color channels. The linear algorithms compute $[r, g, b]^{T}=B v$. The linear methods differ only in the matrix $B$ used to compute the map.

If a method produces colors that are not representable on the display (a coordinate lies outside the interval $[0,1]$ ), clipping (projection) is used. Clipping maps nonrepresentable colors to the surface of the 3-cube. A projection map is linear. 
Region merging occurs when adjacent regions of different colors are mapped to the same anaglyph color. This can affect depth and detail perceived in stereo images ${ }^{1}$. We note that clipping can cause region merging. Ghosting or crosstalk means that one eye can see part of the image for the other eye.

The spectral distributions of the phosphors on CRTs are shown in Figure 1. They have been uniformly scaled so the maximum value of red is 1 . We include the spectral distributions of the primaries for LCD monitors.

The conversion from RGB space to CIE space requires a linear transformation represented by the matrix $C^{3}$. The matrix $C$ for the CRT spectral distributions in Figure 1 is

$$
C=\left[\begin{array}{ccc}
X_{R} & X_{G} & X_{B} \\
Y_{R} & Y_{G} & Y_{B} \\
Z_{R} & Z_{G} & Z_{B}
\end{array}\right]=\left[\begin{array}{ccc}
11.6638 & 8.3959 & 4.65843 \\
7.10807 & 16.6845 & 2.45008 \\
.527874 & 3.79124 & 24.0604
\end{array}\right]
$$

The gamut (on the CIE chromaticity diagram) for the spectral distributions given in Figure 1 is the RGB triangle in Figure 2.

\section{ANAGLYPH FILTERS}

The colors visible through a filter depend on the transmission function $f(\lambda)$ of the filter. The function $f$ specifies the percentages of each visible wavelength $\lambda$ transmitted by the filter. The product of the phosphor spectral distribution with the transmission function gives the spectral distribution of the phosphor as seen through the filter. The matrices $A_{l}$ for the left eye and $A_{r}$ for the right eye convert the resulting filtered colors to CIE coordinates. For the red/cyan glasses, these matrices are

$$
A_{l}=\left[\begin{array}{ccc}
5.42327 & .807004 & .047325 \\
2.70972 & .50201 & .0250529 \\
.0000550941 & .000411221 & .00240686
\end{array}\right], A_{r}=\left[\begin{array}{ccc}
.180431 & 1.6395 & 2.00309 \\
.448214 & 6.31551 & 1.35757 \\
.289201 & 2.3925 & 11.062
\end{array}\right],
$$

Multiplying these matrices by each color in the 3-cube produces a new color solid in CIE space. The resulting gamuts for the left and right eyes are labeled in figure 2. The transmission functions for each filter are shown in figure 3.

The color solids intersect only at the origin and hence there is perfect blocking; there can be no ghosting due to intersection of color solids between the left and right eye views. The color solids in CIE space are shown in Figure 4. They have been scaled by .25 for comparison with the CIE chromaticity triangle. Although CIE space is not a uniform color space, the large differences between the three color solids should be observed. The gamuts are shown in Figure 2.

\section{SAMPLE IMAGES}

For algorithm comparisons we use stereo pair of an Indian mother and her daughter shown in Figure $5^{4}$. They are arranged for cross viewing. The original images were 443 x 389.

The region of comparison is the white rectangle shown in Figure 6 of the left eye view. This is the region [150, $190] \times[200,230]$ in the original scene. Figure 7 shows this 41 x 31 region in the left and right eyes magnified so that individual pixels are visible.

Low intensities of each primary will appear to be blocked by their respective filters. We call this the blocking threshold. For example, red intensities below $60 / 255=.24$ appear to be blocked by the left eye filter.

If the red channels of the pixels in the same area of the anaglyph are not below blocking threshold, the region will be partially seen in both eyes and cause ghosting. We consider the pixel with coordinates $(13,18)$ in the region. The color of this pixel in the right eye view is $[216,197,161] / 255=[0.85,0.77,0.63]$ (rounded) and in the left eye view is $[104,22,18] / 255=[0.41,0.09,0.07]$. An algorithm which chooses a red channel value between the red channel values 
of the left and right eye colors will produce an anaglyph red channel value above the blocking threshold and cause ghosting.

\section{THE PHOTOSHOP ALGORITHM}

\subsection{Algorithm description}

In the original Photoshop algorithm ${ }^{5}$ (PS) the red channel of the left eye view becomes the red channel of the anaglph and vice versa for the blue and green channels of the right eye. This is equivalent to projecting (computing the least squares approximation) the left eye RGB point to the red axis of the RGB cube (setting the G and B channels to zero) and the right eye RGB point to the GB plane (thus setting the red channel to zero). See Figure 8 . The two resulting vectors are added to compute the color of the pixel in the anaglyph. This method is linear. The matrix $B$ is

$$
B=\left[\begin{array}{llllll}
1 & 0 & 0 & 0 & 0 & 0 \\
0 & 0 & 0 & 0 & 1 & 0 \\
0 & 0 & 0 & 0 & 0 & 1
\end{array}\right]
$$

The algorithm ignores the transmission function of the glasses. The computed anaglyph is the same for all filters.

\subsection{Region Merge}

All colors with the same left eye red channel and the same blue/green channels in the right eye view will be mapped to the same color.

\subsection{Ghosting}

The comparison pixel has color $[104,197,161] / 255=[.41, .77, .63]$. The red channel is not below blocking threshold, so there will be ghosting as seen in Figure 9.

\subsection{Conversion to grayscale}

An alternative method converts the left eye image to grayscale first and then projects as described above.

If we use a linear grayscale conversion algorithm (such as the NTSC luminance standard where grayscale $=.299 \mathrm{r}+$ $.587 \mathrm{~g}+.114 \mathrm{~b}[6])$ we premultiply $v$ by a partitioned matrix of the form

where $\mathrm{I}$ is the $3 \times 3$ identity and $\mathrm{G}$ is the matrix

$$
\left[\begin{array}{cc}
G & 0 \\
0 & I
\end{array}\right]
$$

$$
G=\left[\begin{array}{ccc}
\alpha_{1} & \alpha_{2} & \alpha_{3} \\
0 & 1 & 0 \\
0 & 0 & 1
\end{array}\right]
$$

where the $\alpha_{i}{ }^{\prime} S$ are nonnegative, sum to 1, and are the coefficients that convert the red channel to grayscale. We then apply the matrix $B$ above. This gives the new matrix

$$
B=\left[\begin{array}{cccccc}
\alpha_{1} & \alpha_{2} & \alpha_{3} & 0 & 0 & 0 \\
0 & 0 & 0 & 0 & 1 & 0 \\
0 & 0 & 0 & 0 & 0 & 1
\end{array}\right]
$$

No clipping is required. Region merge and loss of detail are still problems since the red channel in the right eye view is ignored. 


\section{THE MIDPOINT ALGORITHM}

\subsection{Algorithm description}

In the midpoint algorithm (MID) we compute in the uniform CIEL*a*b*7 space (Lab) the point $\mathrm{P}$ that minimizes the sum of the distances between the transformed filtered left and filtered right eye color values at a pixel. The midpoint of the line joining the two colors minimizes this sum. We map the left eye color to CIE space using $A_{l}$ and then to Lab space using the Lab transformation. The algorithm is similar for the right eye. We use the D65 ${ }^{7}$ illuminate values for Xn, $\mathrm{Yn}$ and $\mathrm{Zn}$ in the MID map. The results for illuminates A and $\mathrm{C}$ were similar.

We apply the inverse map to return to RGB space. We then multiply by $\mathrm{N}$ where $\mathrm{N}$ is a normalizing diagonal matrix that ensures the [white,white] vertex in the 6-cube is mapped to the white vertex in the 3-cube.

The algorithm is not linear. The images in Lab space of the primary axes for the $A_{l}$ and $A_{r}$ color solids in CIE space are shown in Figure 10. One of the axes for each solid is very short and is difficult to see in the figure.

\subsection{Region Merge}

All colors in the left and right eye color solids with the same midpoint in Lab space are mapped to the same RGB color. The image of the vertices of the 6-cube in using MID are shown in Figure 11. Some vertices lie outside the RGB cube and clipping will be required.

\subsection{Ghosting}

While the MID method uses the filter properties, severe ghosting can occur because the left and right eye views may both have relatively high intensity red (or green/blue) components. An example is given in Figure 12. The color of the comparison pixel is $[109,184,145] / 255=[.43, .72, .57]$.

We note that we also tried the midpoint calculation in CIE space which is a linear algorithm. The results were poor.

\section{THE DUBOIS ALGORITHM}

\subsection{Algorithm Description}

Eric Dubois ${ }^{3}$ suggests least squares (LS) projection in $\boldsymbol{R} \boldsymbol{6}$ to the 3D subspace spanned by the 6 dimensional columns of the partitioned $6 \times 3$ matrix $R$ defined below with right hand side partitioned vector $D$ where the matrix $C$ is the RGB-CIE conversion matrix defined above:

$$
R=\left[\begin{array}{l}
A_{l} \\
A_{r}
\end{array}\right], D=\left[\begin{array}{ll}
C & 0 \\
0 & C
\end{array}\right] v
$$

The projection minimizes the Euclidean length of the vector $R[r, g, b]^{T}-D$, and is the least squares approximation or projection.

The algorithm also uses scaling by a diagonal matrix $N$ as in the midpoint method. The linear map from $\boldsymbol{R} \boldsymbol{6}$ to $\boldsymbol{R} \mathbf{3}$ is therefore $[r, g, b]^{T}=N\left(R^{T} R\right)^{-1} R^{T} D$. The matrix $B$ is

$$
B=\left[\begin{array}{cccccc}
0.4561 & .500484 & .176381 & -.0434706 & -.0879388 & -0.00155529 \\
-.0400822 & -.0378246 & -.0157589 & .378476 & .73364 & -.0184503 \\
-.0152161 & -.0205971 & -.00546856 & -.0721527 & -.112961 & 1.2264
\end{array}\right]
$$

The relationship of the RGB and LS gamuts are shown in Figure 13. The LS gamut contains nonvisible colors in addition to colors not realizable on a CRT.

\subsection{Region Merge}

Interval analysis shows that because the LS color solid in $\boldsymbol{R 3}$ properly contains the RGB cube, clipping will be required. Finding colors that map to the same color that are not a result of clipping is not an easy task. We used the image of the $\boldsymbol{R} \boldsymbol{6}$ vertices as shown in Figure 14. The larger dots are the extreme vertices. 
Figure 15 shows the order of the first 10 vertices of the hypercube mapped to $\boldsymbol{R} 3$. This vertex sequence creates a parallelopiped whose"front" and "back" planes are very close together (.029 Euclidean distance) corresponding to the same left eye color (see Figure 16). This is true for all left eye colors of the form $[\mathrm{x}, \mathrm{y}, \mathrm{z}]$ where $\mathrm{x}, \mathrm{y}$, and $\mathrm{z}$ are 0 or 1 (it is not true for all left eye colors). The quadrilateral corresponding to the projection of all colors of the form [r,g,b,0,u,v], $\mathrm{u}$ and $\mathrm{v}$ in $[0,1]$ is parallel to and overlaps the quadrilateral corresponding to the projection of all colors of the form $[\mathrm{r}, \mathrm{g}, \mathrm{b}, 1, \mathrm{u}, \mathrm{v}], \mathrm{u}, \mathrm{v}$ in $[0,1]$. The region is a bounded by the vertices [r,g,b,0,1,0], [r,g,b,0,1,1], [r,g,b,1,0,0] and $[\mathrm{r}, \mathrm{g}, \mathrm{b}, 1,0,1]$. The first vertex in a plane (in the binary ordering) is the one that occurs on the black/red edge.

Figure 17 shows how the planes are ordered. Note that if we label the planes from 0 to 7 , then plane 0 moves to plane 1 to plane 3 to plane 5 back to plane 2 to plane 4 to plane 6 to plane 7 . Hence there are colors with two different left eye coordinates (and two different right eye coordinates) that will map to the same RGB color (ignoring the clipping that is required for colors outside the RGB cube). Examples, shown in Figure 18, are colors $1=[.904,0$, 0, 1, $.25, .5]$ and colors $2=[0.904107,0,0,0,0.765829,0.48873]$ that have radically different right eye colors. Their LS colors are both [0.344257, $0.516587,0.49911]$.

\subsection{Ghosting}

The comparison pixel in the Dubois LS calculation is $[35,214,158] / 255=[.14, .84, .62]$ which has a red channel value below blocking threshold and no ghosting occurs in this case. The anaglyph image is Figure 19. For the stereo pairs studied, the LS method did not demonstrate ghosting.

\section{CONCLUSIONS AND DIRECTIONS FOR FUTURE RESEARCH}

Linear anaglyph algorithms will always map several different left/right eye colors to the same RGB color. Perhaps nonlinear algorithms exist that avoid region merge. The results appear to show that for color images the MID method produces excellent color and detail but may suffer severe ghosting. An anaglyph produced by the LS method is normally darker with less detail and requires brightening or gamma correction but appears to have no ghosting. Further investigation of the properties of the LS solid in $\boldsymbol{R} \mathbf{3}$ is necessary. The PS method is easy to implement and works well for grayscale images but may also suffer ghosting and poor color representation. Because of stereo and color conflicts it appears that formulating an anaglyph algorithm that always produces good color representation, good detail, no ghosting, and no region merging is not possible.

We speculate that the ability to ray trace the original 3D scene can help produce a better method. Properties of anaglyph filters for display devices also need more investigation.

\section{ACKNOWLEDGEMENTS}

We wish to thank John W. Fuller of Lee Filters, USA for his help in obtaining the transmission functions used in this paper, to Joel Trussell of North Carolina State University for sharing his phosphor and LCD spectral distributions and to Eric Dubois for his help in implementing the LS algorithm.

\section{REFERENCES}

1. D. F. McAllister and Preshant D. Hebbar, "Color Quantization Aspects in Stereopsis", SPIE Proceedings

Stereoscopic Displays and Applications II, Volume 1457, pp. 233-241, SPIE, Bellingham,WA, 1991

2. D. F. McAllister (Ed.), Stereo Computer Graphics and other True 3D Technologies, Princeton U. Press, Princeton, NJ, 1993

3. Eric Dubois, "A Projection Method to Generate Anaglyph Stereo Images," Proc. IEEE Int. Conf. Acoustics Speech Signal Processing, , vol. 3, pp. 1661-1664, IEEE, Salt Lake City, UT, 2001

4. Ray Hannisian, "Los Huicholes," stereo image on http://www.ray3d.com/road.html/

5. Andrew Woods and John Merritt, "Stereoscopic Display Application Issues," (Short Course Notes), EI '02, SPIE, San Jose, California, 2002.

6. Randy Crane, A Simplified Approach to Image Processing, Prentice Hall PTR, Upper Saddle River, NJ, 1997

7. Daniel Malacara, Color Vision and Colorimetry, SPIE Press, Bellingham, WA, 2002 


\section{FIGURES}
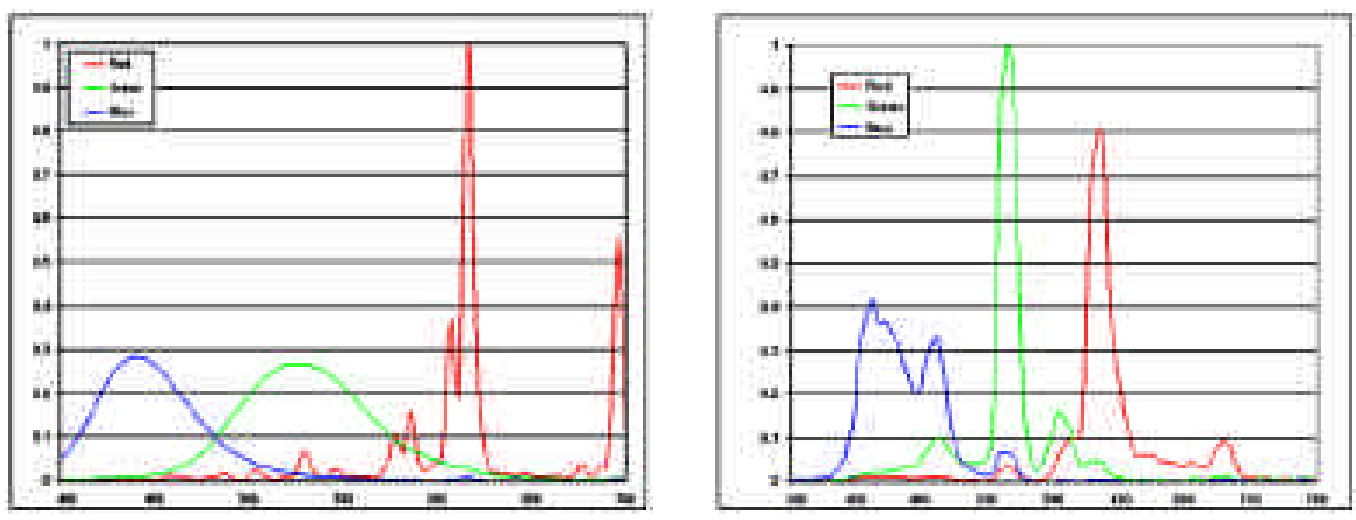

Figure 1: CRT (left) and LCD spectral distributions
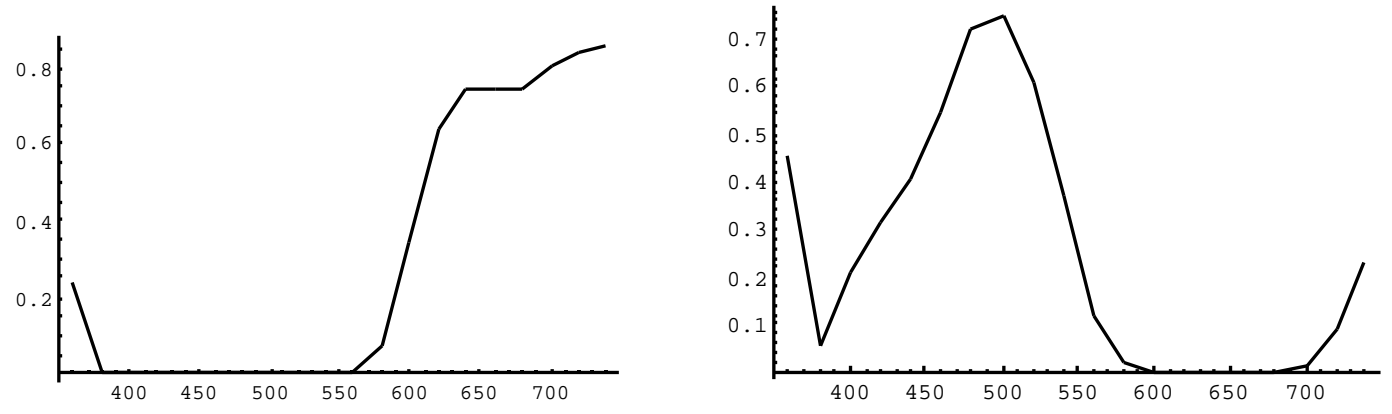

Figure 3: Transmission functions for REEL3D filters 

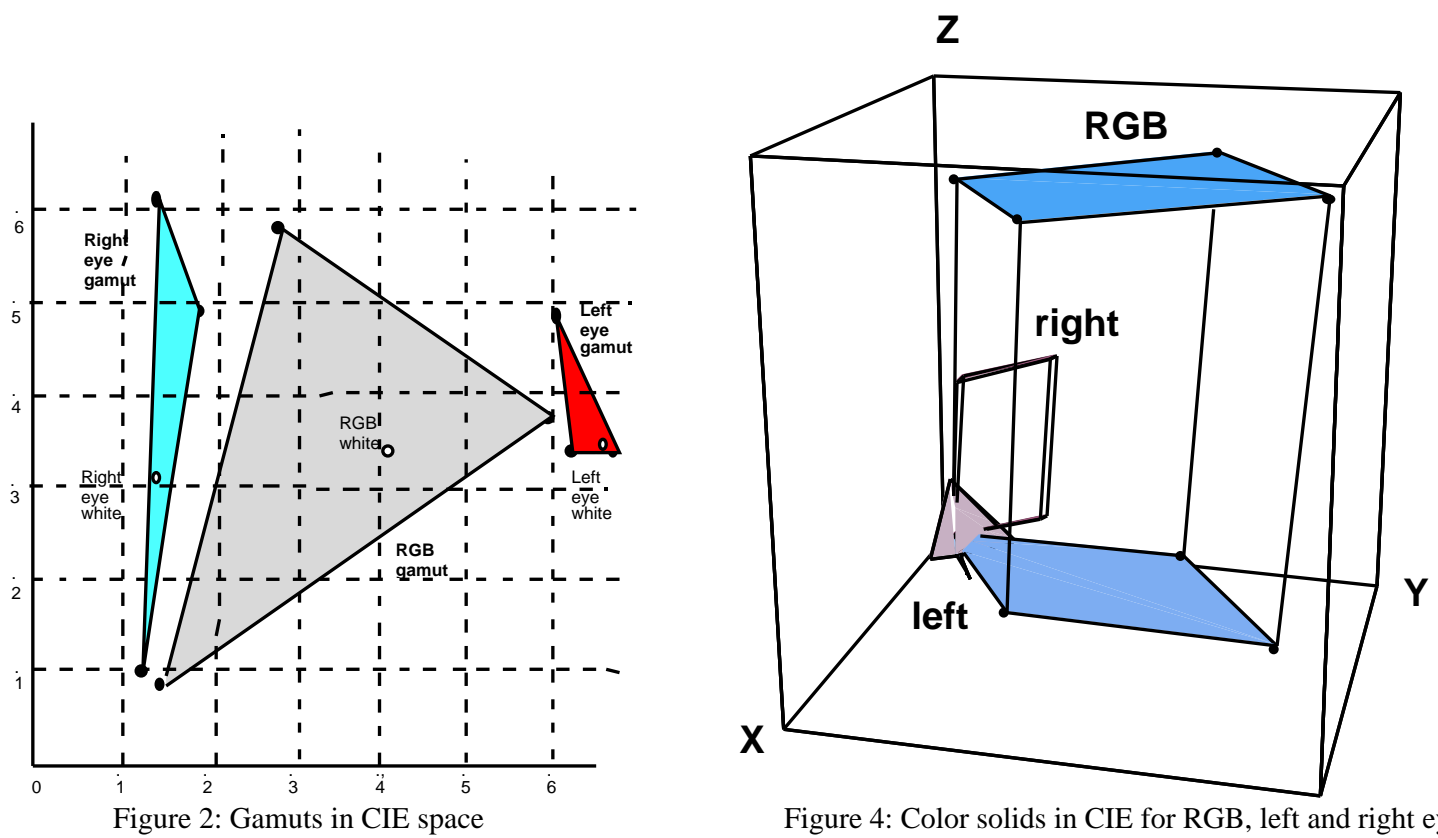

Figure 4: Color solids in CIE for RGB, left and right eyes
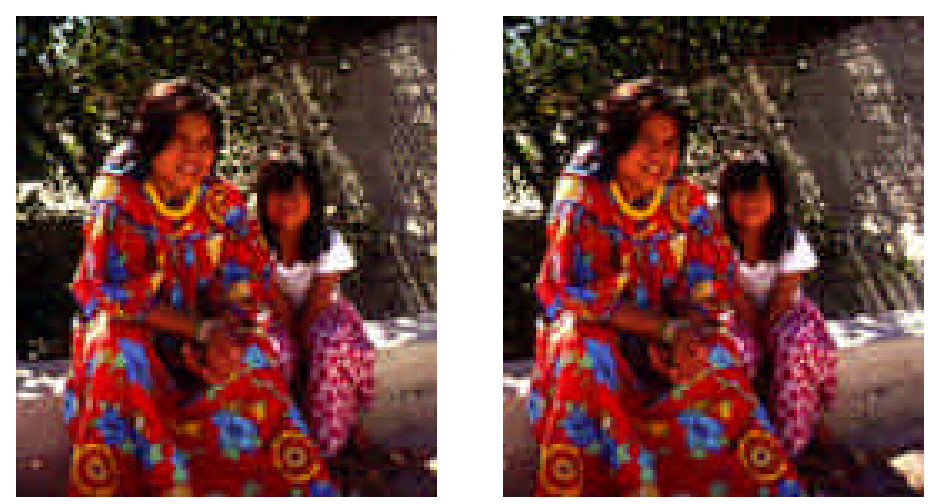

Figure 5: Stereo pair ( use cross viewing)

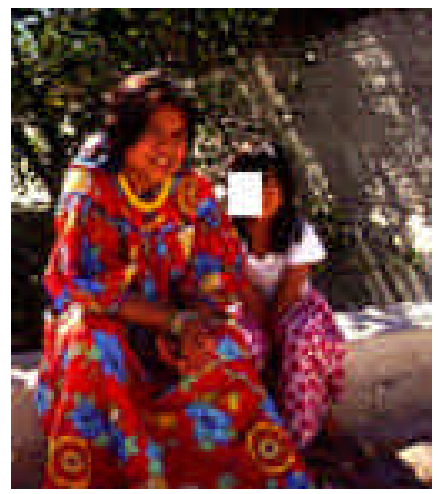

Figure 6: Region in left eye view 


\section{B}
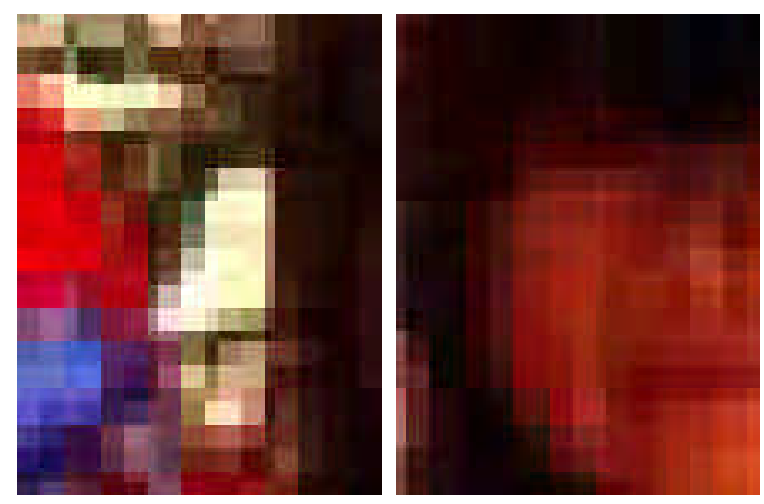

Figure 7: Right and left eye regions upscaled

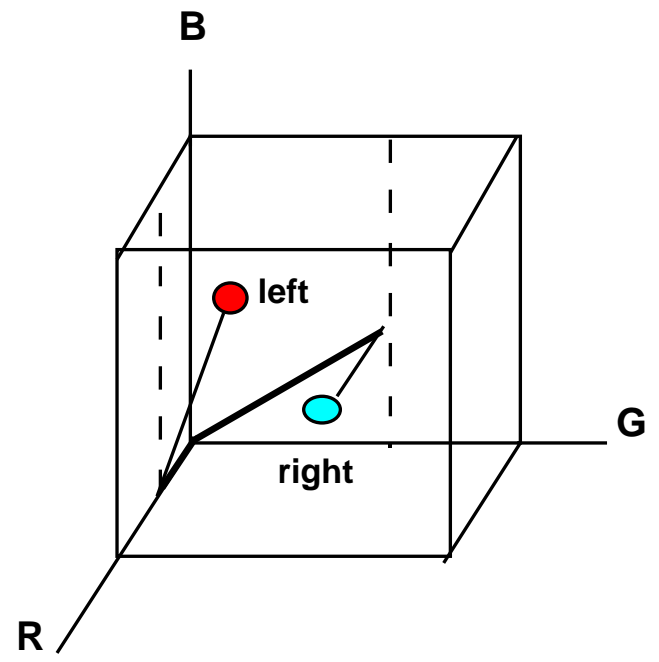

Figure 8: Projection is PS algorithm

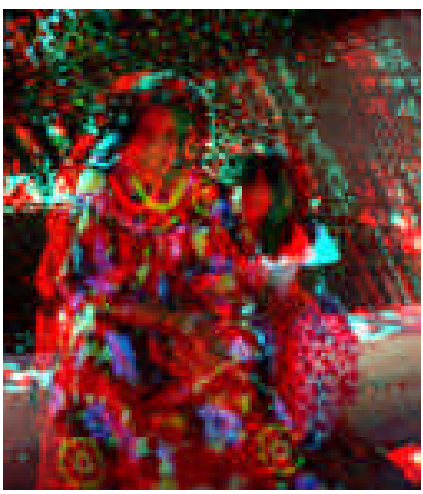

Figure 9: PS anaglyph

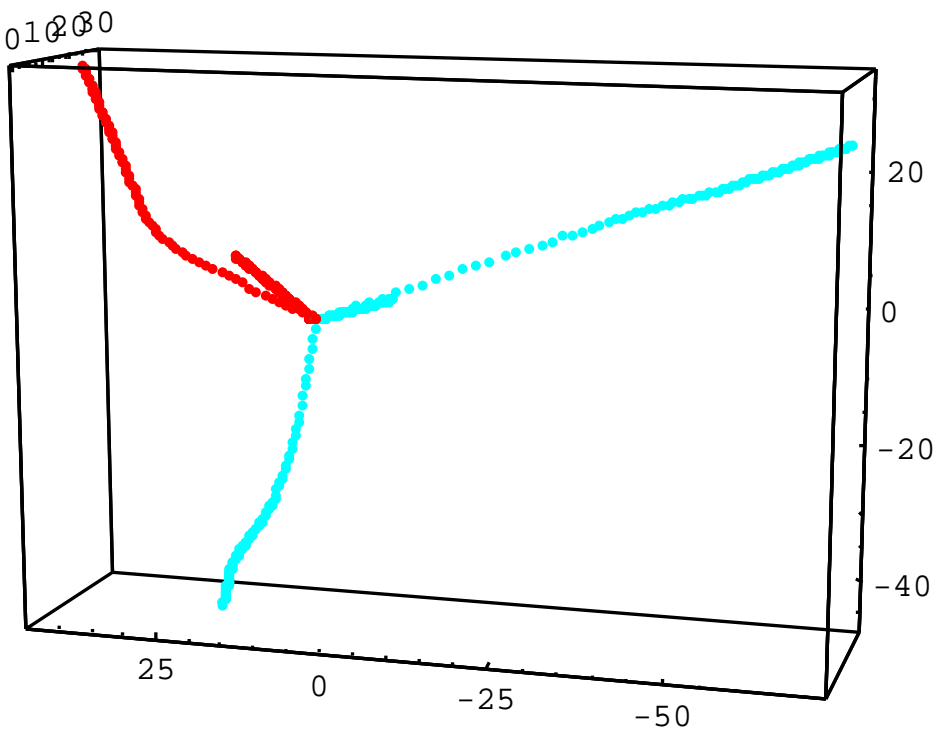

Figure 10: Images in Lab space of the primary axes for the $A_{l}$ and $A_{r}$ color solids 


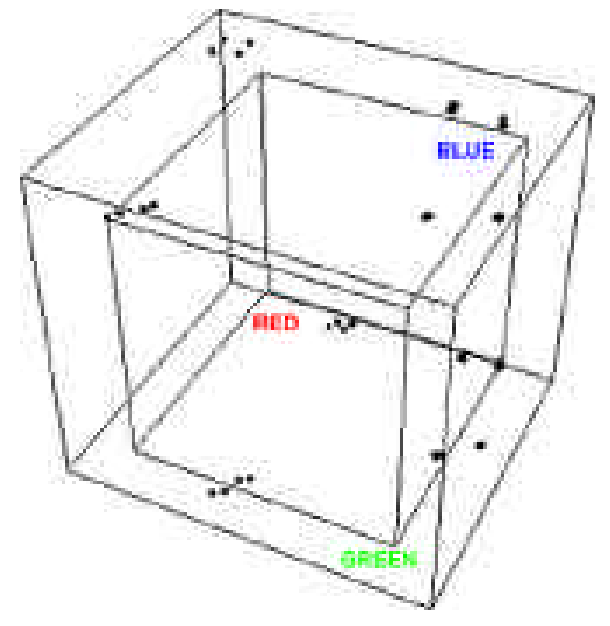

Figure 11: 6-cube vertex plot with 3-cube

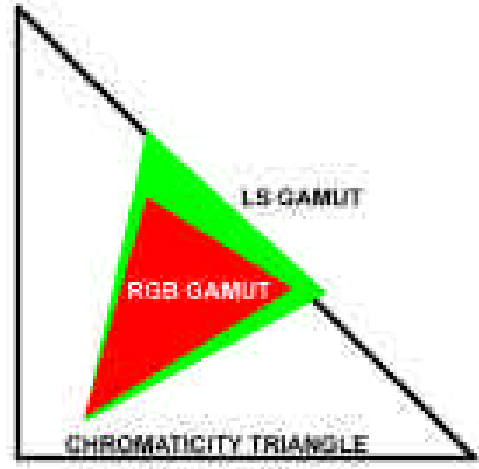

Figure 13: RGB and LS gamuts

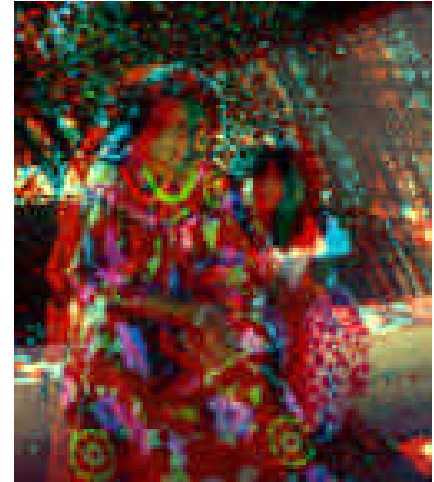

Figure 12: MID anaglyph

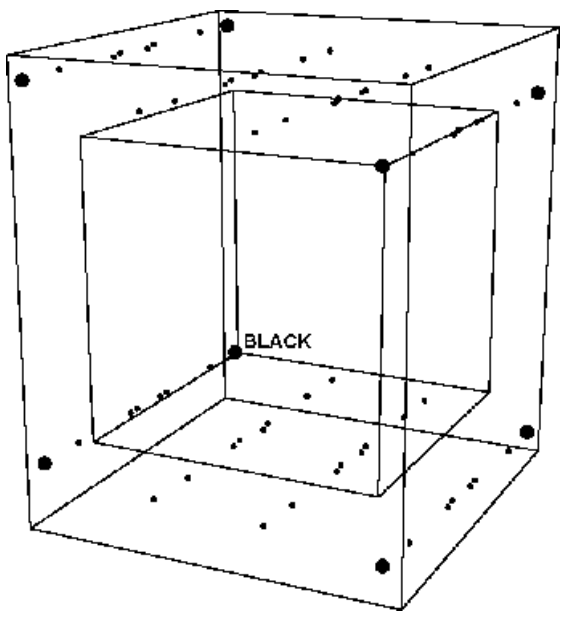

Figure 14: LS Images of 6-cube vertices 

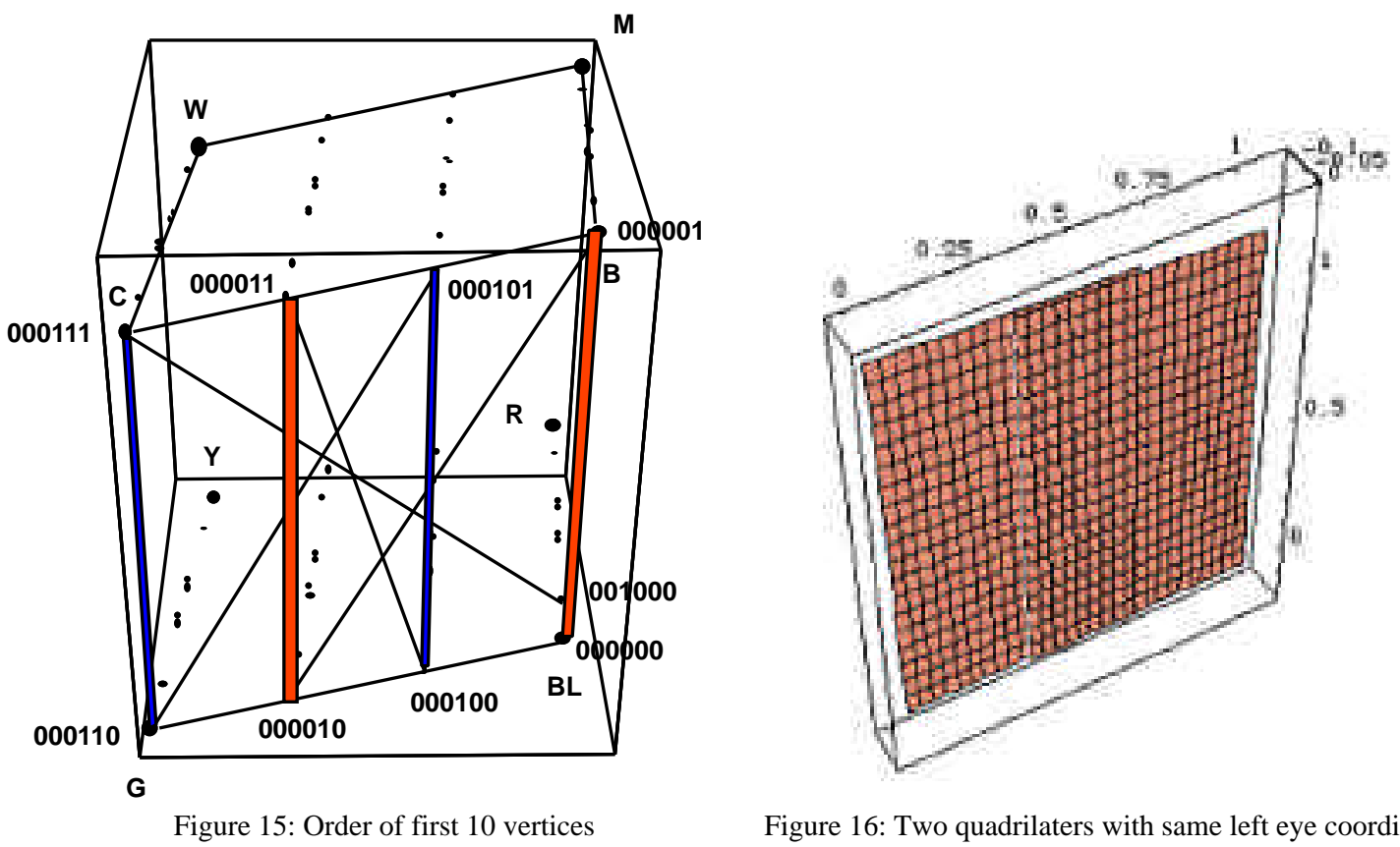

Figure 16: Two quadrilaters with same left eye coordinates

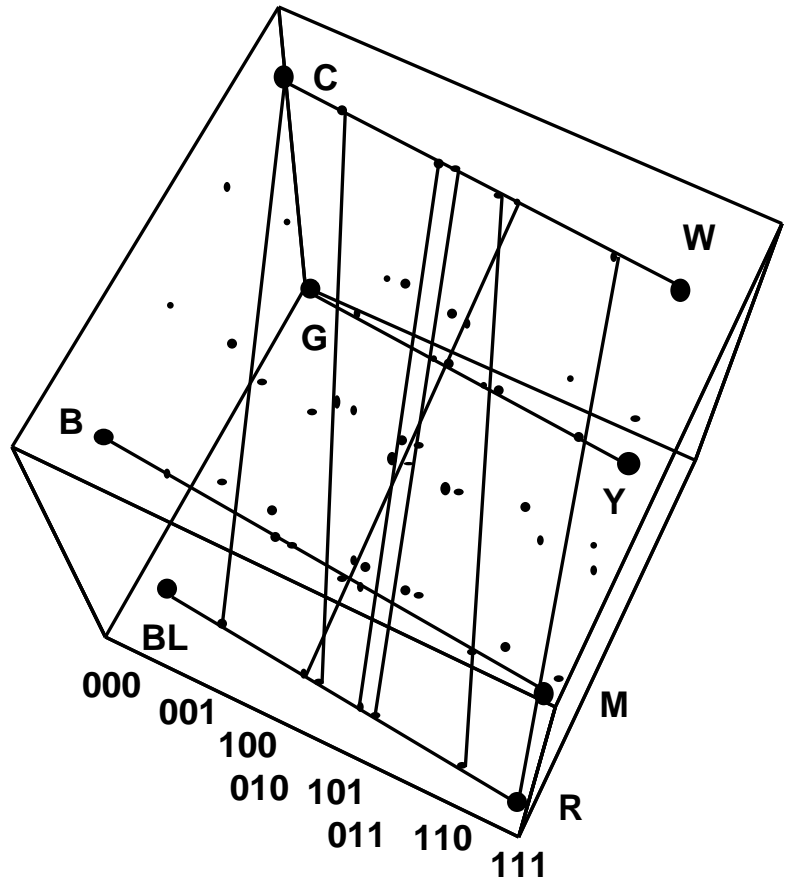

Figure 17: Order of planes of left eye colors 

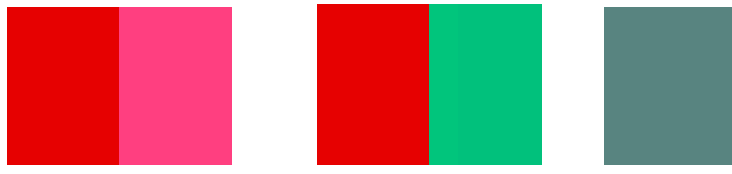

Figure 18: Color pairs which map to same color in LS

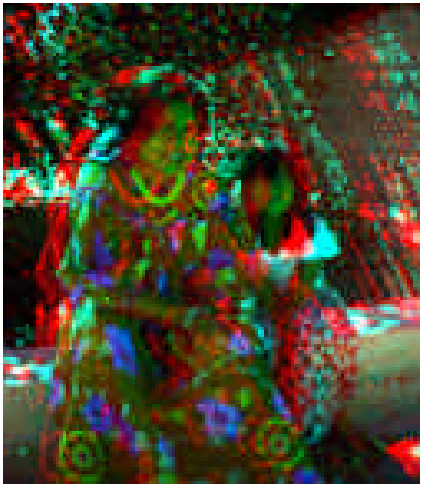

Figure 19: LS anaglyph 